\title{
EZHIP is a specific diagnostic biomarker for posterior fossa ependymomas, group PFA and diffuse midline gliomas H3-WT with EZHIP overexpression
}

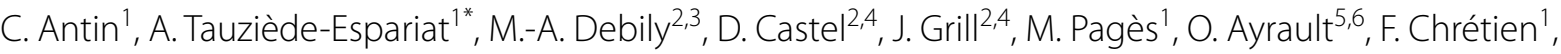 \\ A. Gareton ${ }^{1}$, F. Andreiuolo ${ }^{1}$, E. Lechapt ${ }^{1}$ and P. Varlet ${ }^{1}$
}

In the central nervous system (CNS), the loss of H3K27me3 expression constitutes the hallmark of two different tumor types: diffuse midline glioma (DMG), H3K27-mutant and posterior fossa ependymoma, group PFA (PFA-EPN). In the former, mutations in histone genes (mostly H3F3A K27M and HIST1H3B K27M), present in about $97 \%$ of DMG, inhibit the activity of the Polycomb Repressive Complex 2 (PRC2) methyltransferase [1]. However, these mutations are rare in PFAEPN (accounting for $\approx 4 \%$ of cases) [2]. Recent molecular advances have shown that the Enhancer of Zest Homologs Inhibitory Protein (EZHIP) is overexpressed (due to gene overexpression rather than mutations of the CXorf67 gene) in the large majority of PFA-EPN, and in the remaining cases of DMG showing H3K27me3 loss but lacking histone gene (H3) mutations [1-3]. Indeed, this overexpression mimics the mechanism of histone gene mutations on PRC2 [4]. Usually, the current routine immunohistochemical (IHC) panel in pediatric neuropathology includes H3K27me3 and H3K27M antibodies but not EZHIP. The aim of our study was to evaluate the sensitivity and specificity of the EZHIP biomarker in a large cohort of pediatric tumors, including the most

*Correspondence: a.tauziede-espariat@ghu-paris.fr

1 Department of Neuropathology, GHU Paris-Neurosciences, Sainte-Anne Hospital, 1, rue Cabanis, 75014 Paris, France

Full list of author information is available at the end of the article common tumor types, which arise in the brainstem and the posterior fossa.

We performed IHC for EZHIP using the CXorf67 antibody (Polyclonal; 1:75 dilution; Sigma-Aldrich; Bromma, Sweden) on $3 \mu \mathrm{m}$-thick sections of formalin-fixed, paraffin-embedded tissue samples of these tumors, performed on an Omnis automate. Our study included a total of 311 cases: 298 pediatric tumors of different subtypes (gliomas, embryonal, and ependymal tumors with a morphomolecular diagnosis including DNA-methylation profiling), and 13 posterior fossa ependymomas, Group PFB (for details see Table 1). This series includes some of the tumors previously reported [1]. The IHC were performed on whole sections in 266 cases and on a TMA (tissue microarray) of 45 ependymomas as a validation cohort which included PFA $(n=37)$, H3K27-mutant $(n=2)$ and PFB $(n=6)$. The IHC stainings were scored by three neuropathologists (ATE, PV and EL) independently.

The IHC results (including the validation cohort) are detailed in Table 1. A strong and diffuse EZHIP nuclear staining ( $>90 \%$ of immunopositive tumor cells) was observed in all DMG, H3-wildtype with EZHIP overexpression $(\mathrm{n}=13)$ (Fig. $1 \mathrm{~A}-\mathrm{C})$ and all PFA-EPN $(\mathrm{n}=47)$ (Fig. 2A-C and Additional file 1: Figure S1), except the two EPN, H3K27-mutants (Fig. 2G-I). The majority of germinomas exhibited a strong nuclear immunostaining (94\%, 29/31 cases) associated with a loss of H3K27me3 trimethylation (Fig. 1G-I and Additional file 2: Figure S2). In all other diagnoses, tumor cells were original author(s) and the source, provide a link to the Creative Commons licence, and indicate if changes were made. The images or other third party material in this article are included in the article's Creative Commons licence, unless indicated otherwise in a credit line to the material. If material is not included in the article's Creative Commons licence and your intended use is not permitted by statutory regulation or exceeds the permitted use, you will need to obtain permission directly from the copyright holder. To view a copy of this licence, visit http://creativecommons.org/licenses/by/4.0/. The Creative Commons Public Domain Dedication waiver (http://creativeco mmons.org/publicdomain/zero/1.0/) applies to the data made available in this article, unless otherwise stated in a credit line to the data. 
Table 1 Immunohistochemical results of EZHIP in our series

Diffuse astrocytic and oligodendroglial tumors

Astrocytoma, IDH-mutant, grade 2

$0 / 2(0)$

Oligodendroglioma, IDH-mutant and 1p19q codeleted, grade 2

Epithelioid glioblastoma

Astrocytoma, IDH-mutant, grade 4

DMG, H3K27-mutant

DMG, H3K27-WT with EZHIP overexpression

HGG with MSI

CMMRD

$0 / 6(0)$

Lynch syndrome

$0 / 4(0)$

Diffuse glioma, H3.3 G34-mutant

$0 / 10(0)$

HGG, MYCN-amplified

$0 / 9(0)$

Glioblastoma, IDH-WT

$0 / 10(0)$

Other astrocytic tumors

Pilocytic astrocytoma

High-grade astrocytoma with piloid features

Pleomorphic xanthoastrocytoma with anaplastic features

Ependymal tumors

Myxopapillary ependymoma

Posterior fossa ependymoma

Group PFA

$47 / 47(100)$

Group PFA, H3K27-mutant

$0 / 2(0)$

Group PFB

Supratentorial EPN

YAP1-fusion-positive

C11orf95 fusion-positive

Subependymoma

Neuronal and mixed neuronal-glial tumors

Diffuse leptomeningeal glioneuronal tumor

Tumors of the pineal region

Pineoblastoma

$0 / 10(0)$

Embryonal tumors

Medulloblastoma, group 3

$0 / 5(0)$

Medulloblastoma, group 4

$0 / 5(0)$

Medulloblastoma, SHH-activated

$0 / 10(0)$

Medulloblastoma, WNT-activated

Embryonal tumors with multilayered rosettes, C19MC-amplified

AT/RT

AT/RT MYC

AT/RT SHH

AT/RTTYR

CNS tumor with BCOR internal tandem duplication

CNS high-grade neuroepithelial tumor with MN1 alteration

Germ cell tumors

$A T / R T$ atypical teratoid/rhabdoid tumor, CNS central nervous system, EPN ependymoma, HGG high-grade glioma, MSI microsatellite instability, WT wildtype

a 1 case presents a focal expression of EZHIP (<1\% of tumor cells) 


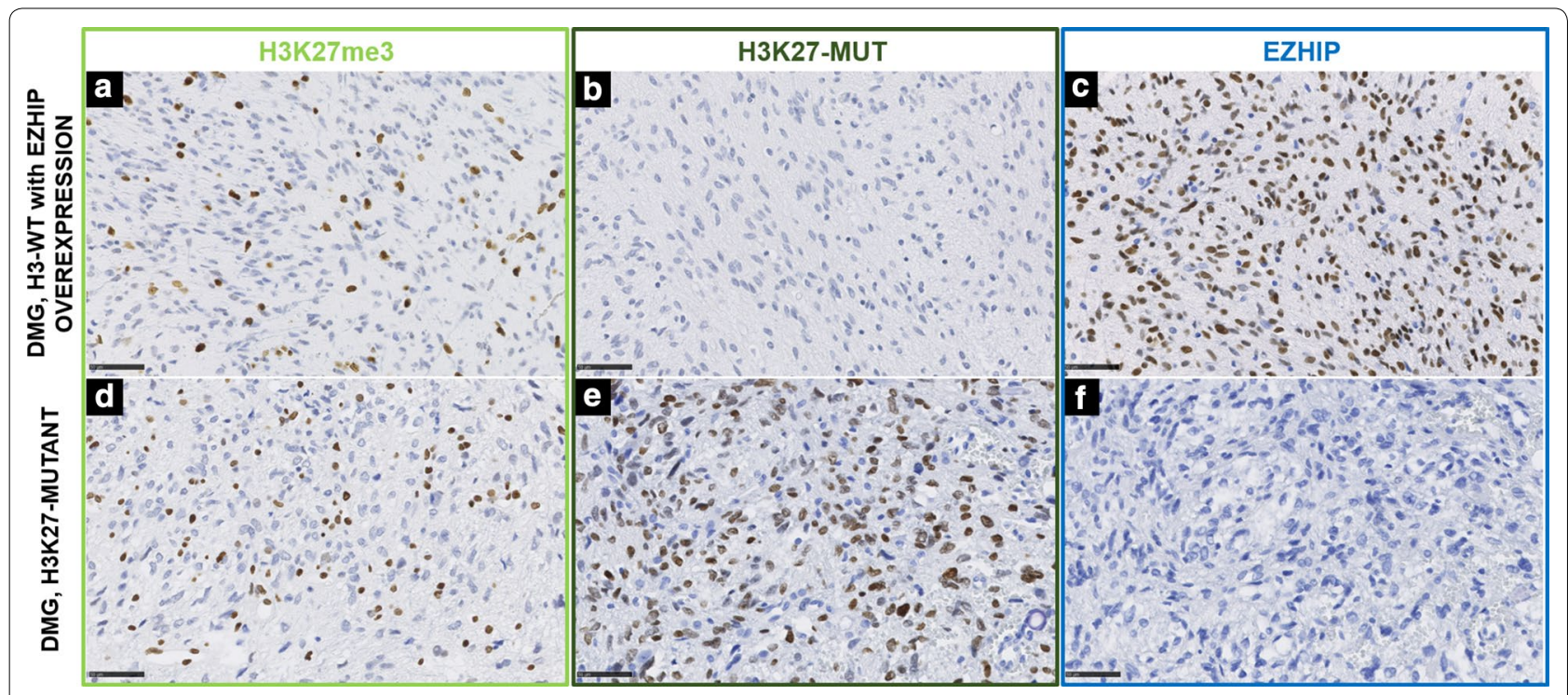

Fig. 1 EZHIP expression in diffuse midline gliomas. A distinct H3K27me3 loss ( $\mathbf{A}$, magnification, $\times 400)$ in one case of diffuse midline glioma with EZHIP overexpression, without expression of H3K27-mutant protein (B, magnification, $\times 400)$ and with strong positive EZHIP expression (C) magnification, $\times 400$ ). A case of diffuse midline glioma, H3K27-mutant with a loss of expression of H3K27me3 (D, magnification, $\times 400$ ), nuclear expression of H3K27-mutant protein ( $\mathbf{E}$, magnification, $\times 400$ ), and without expression of EZHIP $(\mathbf{F}$, magnification, $\times 400)$. DMG diffuse midline glioma, WT wildtype. Black scale bars represent $50 \mu \mathrm{m}$

immunonegative except for two cases: one atypical teratoid/rhabdoid tumor (AT/RT) belonging to the $M Y C$ methylation class and one medulloblastoma, WNT-activated. These two cases exhibited only focal expression ( $<1 \%$ of immunopositive tumor cells) (data not shown). This low protein expression of EZHIP was correlated with a normal level of CXorf67 gene expression at the mRNA level. Thus, the specificity and the sensitivity of the IHC were evaluated as $99 \%$ and $98 \%$ respectively.

This work constitutes the first study of the sensitivity/specificity of EZHIP immunoexpression in a large cohort of CNS tumors. Our results highlighted that nuclear EZHIP expression must be diffuse and strong to be interpreted as overexpressed. Thus, EZHIP IHC constitutes a fast, low-cost and conservative tissueconsuming method to detect CXorf67 overexpression, suitable for small samples (particularly in brainstem biopsies), but also in samples that contain few tumor cells. The IHC may also help to evaluate the quality of resection (surgical limits). Indeed, a nuclear immunopositivity is easier to interpret than the loss of H3K27me3. Our work highlighted the robust specificity of EZHIP staining in all PF ependymomas, group PFA and in all DMG, H3-wildtype with EZHIP overexpression, ruling out the main differential diagnoses encountered in children in the brainstem and in the posterior fossa (Table 1). All germinomas except two exhibited a strong positivity for EZHIP concomitant with a loss of H3K27me3 as published previously [2, 7]. Concerning HGG, MYCN-amplified none of our 9 cases (confirmed by DNA-methylation profiling and previously reported $[5,6])$, were immunopositive, contrarily to a previous study which reported an expression of EZHIP in 13\% of cases [1]. Moreover, this biomarker may represent a diagnostic but also a prognostic tool. Indeed, PFA-EPN were associated with a poorer prognosis than PFBEPN, and patients with DMG overexpressing EZHIP presented a better overall survival compared to DMG, H3K27-mutant [1].

To conclude, we demonstrated that EZHIP IHC is a highly specific and sensitive biomarker for identifying PFA-EPN and DMG, H3-wildtype, with EZHIP overexpression, and should be part of the neuropathologist's routine panel of antibodies. 


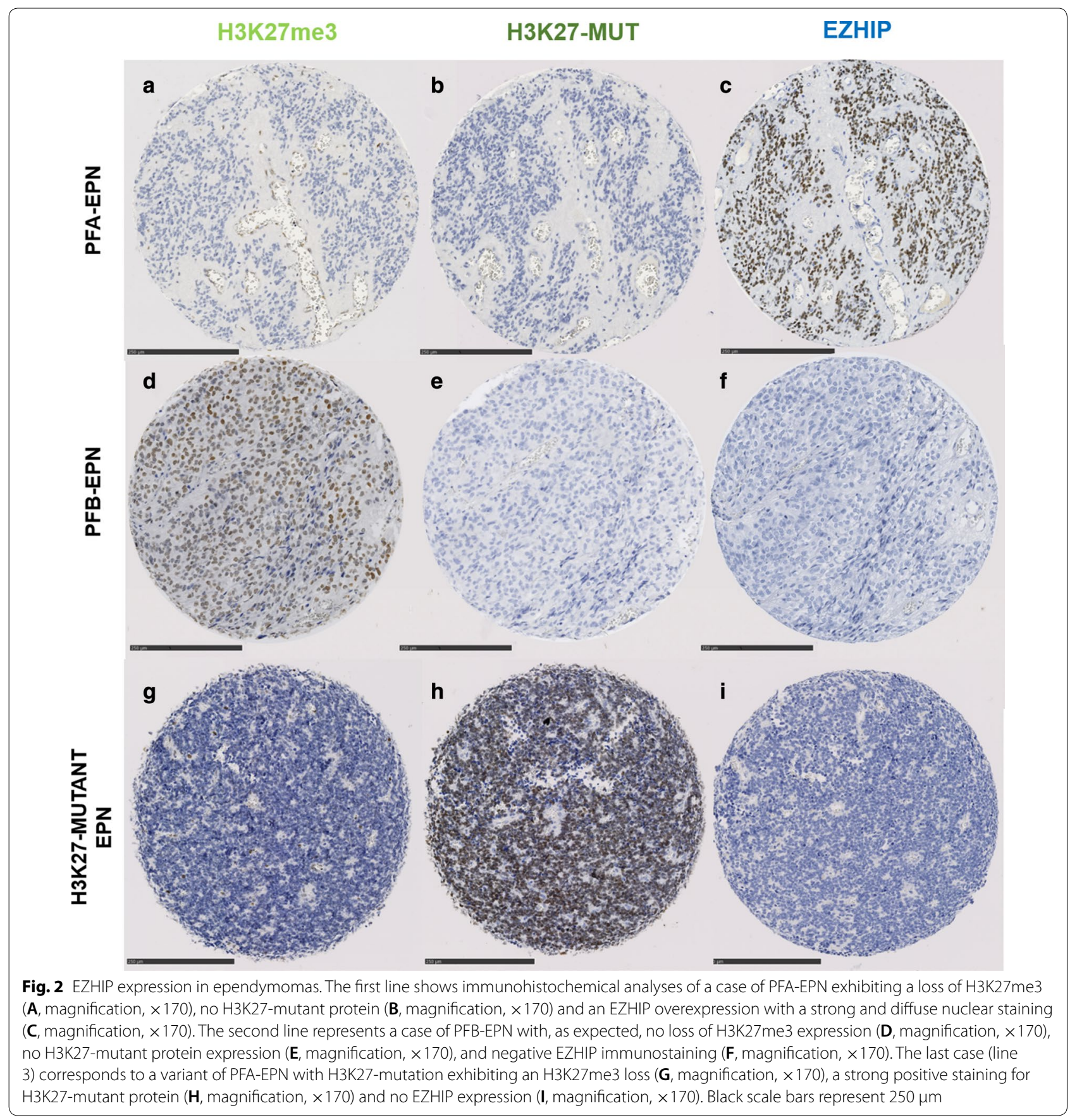




\section{Supplementary information}

Supplementary information accompanies this paper at https://doi. org/10.1186/s40478-020-01056-8.

Additional file 1: Figure S1. EZHIP expression in ependymomas of different grades. The first line shows immunohistochemical analyses of a case of grade 2 PFA-EPN (A, HPS magnification, 400x) exhibiting a loss of H3K27me3 (B, magnification, 400x), and an EZHIP overexpression with a strong and diffuse nuclear staining (C, magnification, 400x). The second line represents a case of grade 3 PFA-EPN with microvascular proliferation and mitoses (white arrowheads) (D, HPS magnification, 400x), with a loss of H3K27me3 expression (E, magnification, 400x), and strong and diffuse EZHIP immunopositivity (F, magnification, 400x). HPS: Hematoxylin Phloxin Saffron. Black scale bars represent $50 \mu \mathrm{m}$.

Additional file 2: Figure S2. EZHIP expression in germinomas. A case of germinoma with H3K27me3 loss (A, magnification, 400x), no expression of H3K27-mutant protein (B, magnification, 400x), and strong and diffuse nuclear immunoexpression of EZHIP (C, magnification, 400x). Black scale bars represent $50 \mu \mathrm{m}$.

\section{Funding}

MAD was supported by a grant from the Kick Cancer/Innovative Therapies for Children with Cancer (ITCC). JG was supported by a grant from Imagine for Margo for the BIOMEDE trial. DC was supported by a grant from L'Etoile de Martin and the Fondation Carrefour "Les Boucles du Coeur".

\section{Competing interests}

The authors declare that they have no conflict of interest directly related to the topic of this article.

\section{Author details}

${ }^{1}$ Department of Neuropathology, GHU Paris-Neurosciences, Sainte-Anne Hospital, 1, rue Cabanis, 75014 Paris, France. ${ }^{2}$ UMR8203, Vectorologie et thérapeutiques anticancéreuses, CNRS, Gustave Roussy, Univ. Paris-Sud, Univ. Paris-Saclay, 94805 Villejuif Cedex, France. ${ }^{3}$ Univ. Evry, Université Paris-Saclay, 91057 Evry Cedex, France. ${ }^{4}$ Department of Pediatric Oncology, Gustave Roussy Institute, Univ. Paris-Sud, Universite Paris-Saclay, 94805 Villejuif, France. ${ }^{5}$ CNRS UMR, INSERM, Institut Curie, PSL Research University, 91898 Orsay, France. ${ }^{6}$ CNRS UMR 3347, INSERM U1021, Université Paris Sud, Université Paris-Saclay, 91898 Orsay, France.
Received: 19 August 2020 Accepted: 10 October 2020

Published online: 05 November 2020

\section{References}

1. Castel D, Kergrohen T, Tauziède-Espariat A, Mackay A, Ghermaoui S, Lechapt E et al (2020) Histone H3 wild-type DIPG/DMG overexpressing EZHIP extend the spectrum diffuse midline gliomas with PRC2 inhibition beyond H3-K27M mutation. Acta Neuropathol (Berl) 139:1109-1113

2. Pajtler KW, Wen J, Sill M, Lin T, Orisme W, Tang B et al (2018) Molecular heterogeneity and CXorf67 alterations in posterior fossa group A (PFA) ependymomas. Acta Neuropathol (Berl) 136:211-226

3. Pratt D, Quezado M, Abdullaev Z, Hawes D, Yang F, Garton HJL et al (2020) Diffuse intrinsic pontine glioma-like tumor with EZHIP expression and molecular features of PFA ependymoma. Acta Neuropathol Commun 8:37

4. Hübner J-M, Müller T, Papageorgiou DN, Mauermann M, Krijgsveld J, Russell RB et al (2019) EZHIP/CXorf67 mimics K27M mutated oncohistones and functions as an intrinsic inhibitor of PRC2 function in aggressive posterior fossa ependymoma. Neuro-Oncol 21:878-889

5. Tauziède-Espariat A, Debily M-A, Castel D, Grill J, Puget S, Sabel M et al (2019) An integrative radiological, histopathological and molecular analysis of pediatric pontine histone-wildtype glioma with MYCN amplification (HGG-MYCN). Acta Neuropathol Commun 7:87

6. Tauziède-Espariat A, Debily M-A, Castel D, Grill J, Puget S, Roux A et al (2020) The pediatric supratentorial MYCN-amplified high-grade gliomas methylation class presents the same radiological, histopathological and molecular features as their pontine counterparts. Acta Neuropathol Commun 8:104

7. Ragazzini R, Pérez-Palacios R, Baymaz IH, Diop S, Ancelin K, Zielinski D et al (2019) EZHIP constrains Polycomb Repressive Complex 2 activity in germ cells. Nat Commun 10:3858

\section{Publisher's Note}

Springer Nature remains neutral with regard to jurisdictional claims in published maps and institutional affiliations.

Ready to submit your research? Choose BMC and benefit from:

- fast, convenient online submission

- thorough peer review by experienced researchers in your field

- rapid publication on acceptance

- support for research data, including large and complex data types

- gold Open Access which fosters wider collaboration and increased citations

- maximum visibility for your research: over 100M website views per year

At $\mathrm{BMC}$, research is always in progress.

Learn more biomedcentral.com/submissions 